\title{
Human Body and Smart Objects
}

\author{
Amon Rapp \\ University of Torino \\ Torino, 10149, Italy \\ amon.rapp@gmail.com

\section{Federica Cena} \\ University of Torino \\ Torino, 10149, Italy \\ cena@di.unito.it

\section{Dize Hilviu} \\ University of Torino \\ Torino, 10123, Italy \\ dize.hilviu@gmail.com
}

\author{
Maurizio Tirassa \\ University of Torino \\ Torino, 10123, Italy \\ maurizio.tirassa@unito.it
}

Permission to make digital or hard copies of all or part of this work for personal or classroom use is granted without fee provided that copies are not made or distributed for profit or commercial advantage and that copies bear this notice and the full citation on the first page. Copyrights for components of this work owned by others than ACM must be honored. Abstracting with credit is permitted. To copy otherwise, or republish, to post on servers or to redistribute to lists, requires prior specific permission and/or a fee. Request permissions from

Permissions@acm.org.

Ubicomp/ISWC 15 Adjunct, September 7-11, 2015, Osaka, Japan

Copyright 2015 (c) ACM 978-1-4503-3575-1/15/09...\$15.00.

http://dx.doi.org/10.1145/2800835.2806204

\begin{abstract}
Smart Objects embed computational capabilities in everyday objects opening opportunities for designing new forms of interaction based on the user's bodily experience. In this work we explore how these devices can exploit their physicality to enrich the interaction between humans and computers.
\end{abstract}

\section{Author Keywords}

Embodied interaction; Smart Objects; Affordances.

\section{ACM Classification Keywords}

H.5.m. Information interfaces and presentation (e.g., $\mathrm{HCI}$ ): Miscellaneous.

\section{Introduction}

Smart Objects are the next generation of ICT systems, embedded in everyday objects, interconnected through the web and endowed with data gathering functions. They can be used to track a variety of user data, from physical to psychological states, from behaviors to habits. Moreover, they can gather environmental or contextual information. Thus, the user's body itself becomes a potentially infinite source of information, leaving a plethora of traces about the user's behaviors and habits. Beside this, the body becomes also a means for interacting with these technologies embedded in objects and environments. One of the 
characteristics of human beings, in fact, is that they seamlessly incorporate the objects they use into bodily practices to the point where individuals perceive these artifacts as extensions of themselves, acting through the objects rather than on them [4]. By allowing users to leverage the physical affordances that everyday objects offer to them, these technologies create new forms of natural interaction based on the knowledge wired in the human body. These can be a lot richer than those offered by the traditional ways of interacting with computers. With keyboard and mouse, for example, we use our bodies in the same manner for a plethora of different tasks like writing texts, communicating with friends, editing images, playing with games, or doing anything else that we may want computation for [11]. Furthermore, interaction with computers is for the most part deliberate and planned, and it requires dedicated cognitive resources, first and foremost attention and memory. Smart objects, instead, allow to transfer the richness of the interaction with everyday objects to the context of digital information and eventually enhance it. We will explore this perspective in the following, by outlining how Smart Objects can exploit the possibilities offered by their peculiarities to create new forms of interaction that leverages the human body capabilities.

\section{Interacting with the body}

Research in Smart Objects prefigures a future when a variety of data could be automatically detected by everyday objects. For example, FoodBoard [15], an instrumented chopping board, uses optical fibers and camera imaging to identify and track ingredients during food preparation on its surface. HealthChair [8] is a chair that detects heart and respiratory rate from its armrests and backrests respectively, making possible an implicit health sensing that requires no additional effort by users. Chigira et al. [2] developed a glass with sensing capabilities that enables unobtrusive hearth rate monitoring during beverage consumption. All these kinds of device can continuously absorb information related to the user's body while she is carrying out her daily activities. Moreover, the user can interact with them by using the skills she already employs for interacting with everyday objects.

However, Smart Objects, thanks to the computational capabilities that are embedded in them, have available a variety of supplementary potentialities that can change the way in which we interact with technology through our bodies.

Within $\mathrm{HCI}$, the role of the body in interacting with computers has been widely explored, in connection with the concepts of embodiment and embodied cognition. Embodied cognition is a heterogeneous movement in cognitive sciences that focuses on a complex "mind-inthe-body, body-in-the-mind" approach instead of the merely syntactic (and therefore devoid of meaning), isolated, disembodied "methodological solipsism" advocated by so-called classical cognitive science [12, 17]. The idea is that the mind should be understood as part (possibly, the conscious part) of a physical body, which it shapes and by which it is shaped. It is this complex that interacts with the world, since we have evolved from creatures whose neural systems were devoted mainly to perceptual and motor processing $[16,18]$. Drawing on Merleau-Ponty's work [14], Dreyfus [4] highlights three different meanings of embodiment: the physical embodiment of a human subject, the set of bodily skills that she has developed and the cultural skills that she gains from the cultural world in which she is situated. Dourish defines 
embodied interaction as "the creation, manipulation, and sharing of meaning through engaged interaction with artifacts" [3]. He notes how the field of tangible computing capitalizes on an array of skills which were previously unexplored in $\mathrm{HCI}$, like the physical skills that we enact in interacting with the real world. From this perspective, tangibles can exploit the natural human skills to provide a more direct and variegate interaction with computational devices. In fact, while traditional GUIs employ the same "abstract", expressly learned, and therefore comparatively unnatural bodily actions for a variety of tasks, tangible interfaces can assign dedicated actions to different functions relying on the motor or embodied memories that are involved when we deal with real world activities [11]. Memory itself, in fact, has been proposed to be not a repository of well-defined and well-structured abstract symbols, but as the continuous, embodied capability to reconceptualize one's state and performance in the situation [7].

However, we think that current explorations of embodiment carried out in connection with tangible interfaces do not address all the opportunities that Smart Objects open for the use of the whole body in interaction with technological devices. Smart Objects, although maintaining the appearances of the common everyday objects, can in fact be enhanced with further communicative and interactive capabilities that can involve body and the embodied cognition in different ways than before. A notion that can help us to understand their novelty is that of affordance. Gibson [6] illustrated how objects naturally offer a set of functionalities, or action possibilities, to specific types of individuals: the affordances of an object, then, invite such individuals to act on it in a specific manner.
Affordances offered by Smart Objects actually do belong to the physical world of everyday life: they invite users to physically act on them, by employing the same bodily skills they use for interacting with the equivalent everyday objects they represent (we can interact with a smart chair in the same way we do with a "normal chair"). Nevertheless, at the same time these affordances can change dynamically thanks to the computational capabilities embedded in them. Recently, Smart Objects affordances have been explored in relation to the notion of ambiguity and the cognitive dissonance they may provoke [13]. However, the more pressing question is how we can integrate the physical affordances of everyday objects with some kind of new enhanced affordances that can enrich and change the interaction. As long as the materiality of the objects wil become more and more computationally transformable and reconfigurable, as envisioned by Ishii [5], these new smart physical affordances will play a more central role in Human-Computer Interaction. We will illustrate this point in the following paragraph through a design concept.

On the psychological side, the backbone of a theoretical analysis of how smart objects may be incorporated in the user's flows of activity should be provided by phenomenology (e.g., [12]), studies of the perception/action cycle (e.g., [6], [7]), and studies of distributed cognition (e.g., [9]).

\section{Interacting with a Smart Object}

We imagine a novel device fully integrated in the everyday life of people, allowing a continuous and transparent gathering of a plethora of user behavioral data related to sedentary behaviors. It could detect user's movements, sleep, postures, physical activities 
by simply being draped on her body. This smart object can be imagined as an enhanced article of clothing that user can take with her while moving through different contexts. It can adapt itself to the positions that the user's body assumes and to the different contexts in which it is used: for example it can monitor the user's sleep behavior when she goes to sleep, or her posture while she is sitting on a chair.

The user can interact with this Smart Object by using its physical affordances that refer to the bodily skills she commonly employs when she interacts with normal clothes: she can wear it, bend it, use it to cover herself or other objects as if it were an everyday object. However, by having the capabilities of changing its physical properties in response to the user's actions, the Smart Object could widen the possibilities of physical interactions by means of its enhanced affordances. For example, it could modify its stiffness according to features of the context in which it is situated or the data it gathered: this could also change the opportunities that the user would have to physically act on or with it.

Furthermore, the Smart Object can also use its enhanced affordances, such as changes in the colors of its material, or different modulations of its surface temperature to continuously keep in contact with the user's body. These affordances can exploit the possibilities of all the bodily senses by which the user normally interacts with the environment. This way, it could actively interact with the user not through linguistic or iconic messages, but via evocative, indirect and continuous affordances using different sensorial channels, such as lights, sounds, vibrations, heat, which can act directly upon the user's body. This modality of communication can be described with the notion of "phatic cue". It represents an affordance that establishes a continuous "phatic communication" [10] between the user and the Smart Object, leveraging the physical features of the object, e.g. the variation of its color, temperature, sound emission, to communicate a change in its internal status or in the user's state [1].

\section{Conclusion}

In this paper we explored how Smart Objects may open new spaces for exploiting bodily interaction with computers, presenting the concept of an innovative Smart Object as an example of these ideas. As a future work, we think that a deeper exploration of the notion of affordance applied to Smart Objects is essential to understand the potentialities of these enhanced everyday objects.

\section{References}

1. Federica Cena, Amon Rapp, Alessandro Marcengo, Adelina Brizio, Dize Hilviu, and Maurizio Tirassa. 2014. The role of affordance in cyber-physical systems for behavioral change. In Proceedings of The First International Conference on Cognitive Internet of Things Technologies (COIOTE '14).

2. Hiroshi Chigira, Masayuki Ihara, Minoru Kobayashi, Akimichi Tanaka, and Tomohiro Tanaka. 2014. Heart rate monitoring through the surface of a drinkware. In Proceedings of the 2014 ACM International Joint Conference on Pervasive and Ubiquitous Computing (UbiComp '14), 685-689. http://doi.acm.org/10.1145/2632048.2632101

3. Paul Dourish. 2001. Where the action is: The foundations of embodied interaction. MIT Press, Cambridge, MA.

4. Hubert L. Dreyfus. 1992. Being-in-the-world. MIT Press, Cambridge, MA. 
5. Hiroshi Ishii, Dávid Lakatos, Leonardo Bonanni, and Jean-Baptiste Labrune. 2012. Radical atoms:

beyond tangible bits, toward transformable materials. interactions 19, 1 (January 2012), 3851. http://doi.acm.org/10.1145/2065327.2065337

6. James J. Gibson. 1977. The theory of affordances. In Perceiving, acting, and knowing: Toward an ecological psychology, Robert E. Shaw \& John Bransford (Eds.). Lawrence Erlbaum Associates Inc., Hillsdale, NJ, 67-82.

7. Arthur M. Glenberg. 1997. What memory is for. Behavioral and Brain Sciences, 20: 1-55.

8. Erin Griffiths, T. Scott Saponas, and A. J. Bernheim Brush. 2014. Health chair: Implicitly sensing heart and respiratory rate. In Proceedings of the 2014 ACM International Joint Conference on Pervasive and Ubiquitous Computing (UbiComp '14). ACM, New York, NY, USA, 661-671. http://doi.acm.org/10.1145/2632048.2632099

9. Edwin Hutchins. 2010. Cognitive ecology. Topics in Cognitive Science, 2, 705-715.

10. Roman Jakobson. 1960. Linguistics and poetics. In Style in language, Thomas A. Sebeok (Ed.). John Wiley, New York, London, UK, 350-385.

11. Scott R. Klemmer, Björn Hartmann, and Leila Takayama. 2006. How bodies matter: Five themes for interaction design. In Proceedings of the 6th Conference on Designing Interactive Systems (DIS '06), 140-149. http://doi.acm.org/10.1145/1142405.1142429

12. Valeria Manera and Maurizio Tirassa. 2010. Cognitive science. In The pragmatics encyclopedia, Louise Cummings (Ed.). Routledge, London and New York, 55-58.

13. Assunta Matassa and Rossana Simeoni. 2014 Eliciting Affordances for Smart Objects in IoT Era. In proceedings of COIOTE 2014. In Internet of Things. User-Centric IoT. Lecture Notes of the Institute for Computer Sciences, Social Informatics and Telecommunications Engineering Volume 150, 2015, 77-81.

14. Maurice Merleau-Ponty. 1962. Phenomenology of perception. Routledge \& Kegan Paul, London, UK.

15. Cuong Pham, Daniel Jackson, Johannes Schoening, Tom Bartindale, Thomas Ploetz, and Patrick Olivier. 2013. FoodBoard: Surface contact imaging for food recognition. In Proceedings of the 2013 ACM International Joint Conference on Pervasive and Ubiquitous Computing (UbiComp '13), 749-752. http://doi.acm.org/10.1145/2493432.2493522

16. Maurizio Tirassa, Antonella Carassa, and Giuliano Geminiani. 2000. A theoretical framework for the study of spatial cognition. In Spatial cognition. Foundations and applications, Seán Ó Nualláin (Ed.). Benjamins, Amsterdam and Philadelphia, 1931.

17. Maurizio Tirassa, and Marianna Vallana. 2010. Representation and computation. In The pragmatics encyclopedia, Louise Cummings (Ed.). Routledge, London and New York, 399-402.

18. Margaret Wilson. 2001. Six views of embodied cognition. Psychonomic Bulletin \& Review, 9, 4: 625-636. 